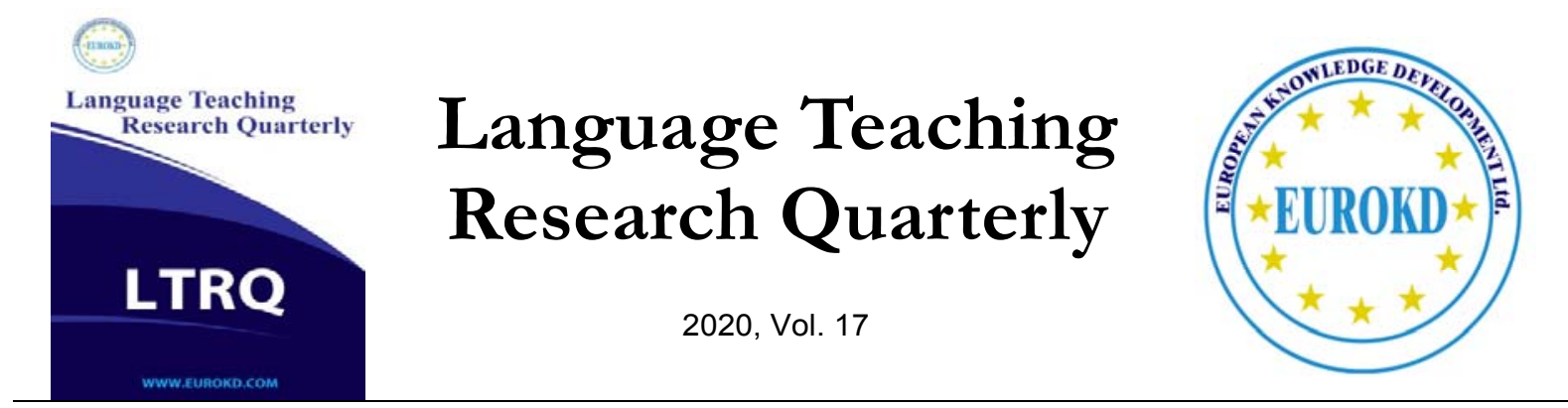

Preface to the Special Issue

\title{
Preface to the Special Issue: Interculturalism in Second Language Teaching
}

\author{
Guest Editor \\ Isaak Papadopoulos, European University Cypruc, Greece \\ I.Papadopoulos@external.euc.ac.cy
}

This is the edited volume related to the broad but interesting thematic area of "Interculturalism in Second Language Teaching". The multilingual and multicultural panorama of the current world intensifies the need for placing interculturalism at the center of the research and teaching activity. Especially, in classrooms with linguistic and cultural diversity, interculturalism should be promoted as a source of teaching and learning while important research has been carried out towards investigating the contribution of interculturalism to second language teaching practices.

Within this context of linguistic and cultural diversity, students are encouraged to develop their intercultural competences, so that they can be prepared to act in a diverse world. In particular, students should develop their intercultural sensitivity and awareness, which can help them to realise and appreciate the differentiations among people from different cultures and achieve mutual understanding. In this way, they are aimed to also develop their intercultural communication strategies, which are proposed by the Council of Europe as important mechanisms for facilitating the communication and co-existence of people in multilingual and multicultural environment and they can be of great help for students' interactions and exchanges in a classroom. Students need to develop such competences of communication, participation and interaction which can facilitate their work and activity within a classroom with students from different countries, while such competences can be extremely important for them later in life. 
This volume has collected important papers from scholars dealing with issues of intercultural and they can contribute to the theoretical and applied scientific knowledge from different perspectives.

This $1^{\text {st }}$ Paper "Promoting intercultural communicative competence through CLIL in Greek primary education" which is authored by Fotini Lagou and Vasilios Zorbas, is highly related to the promotion of interculturalism in language classrooms. More specifically, the authors/researchers aspired to investigate both the intercultural practices of EFL teachers in Greek schools and the extent to which Content and Language Integrated Learning (CLIL) contributes to fostering intercultural competences to students. Among their findings, the authors highlight that "although primary teachers in Greece lack the necessary disciplinary knowledge to promote ICC, they still remain motivated individuals, willing to go the extra mile in order to improve their expertise", which is a really encouraging aspect and calls for more training programs offered to them.

The $2^{\text {nd }}$ Paper "From observation of teaching practices to mentoring and teacher development in a multicultural setting: A case study" has been written by Vassilis Parathyras and Vasilios Zorbas. The researchers/authors investigated whether teacher mentoring could be developed and implemented in the Greek EFL multicultural teaching context, through observation as well as the extent to which such a programme could contribute to the teacher (observee) or even mentor (observer) development. Among their findings, it is worth mentioning their proposal for a direct observation form which can be used for observing teaching practices in a multicultural setting in combination with face-to-face sessions for the provision of feedback.

The 3rd Paper "Applying Gustav Heckmann's version of the Socratic Method and the Hawaiian Talk Therapy to students with Developmental Disabilities: an Intercultural Case Study" has been authored by Alexandros Colombos. This paper describes a case study of a Greek-American young adult and a New York City non-profit Day Habilitation consumer diagnosed with various developmental disabilities. In this paper, Gustav Heckman's version of Socratic Method called Socratic Enquiry used in individual sessions (one-on-one) for teaching Greek as a Second Language and English Reading and Writing while social/behavioral skills group therapy sessions of five consumers where Hawaiian Talk Story was used within the context of the methods used for therapeutic and educational interventions.

The $4^{\text {th }}$ Paper "Interculturalism in Content and Language Integrated Learning Classes: research perspectives from European policy to Greek reality" has been written by Georgios Sakellariou and Isaak Papadopoulos. The authors of the paper attempted to cover theoretical review on CLIL and its development, placing special emphasis on the priorities of the European Council within the context of promoting multilingualism in Europe, promoting CLIL as an effective approach. Moreover, they have included detailed references to organizations promoting the implementation of CLIL across Europe, underlining the CLIL position and implementation in the Greek context. 
The $5^{\text {th }}$ Paper “Intercultural Story-Based Framework in Young Language Learners' Classrooms: from alternative teaching to alternative assessment" has been written by Bisiri Effrosyni. In this paper, the researcher/author attempts the presentation of a story-based framework for young learners at beginner's level pre-A1 of the CEFR through using intercultural stories. Among her priorities, intercultural stories can enhance young learners' creativity and develop their four language skills holistically. She also proposes alternative assessment tools to meet the needs of an alternative classroom environment like the one with linguistic and cultural diversity.

Given the above, we hope that this volume will be of help and interest to all the readers dealing with second language education classrooms. Through the papers' combination of theoretical and methodological/practical knowledge, teachers may find interesting ideas and follow principles and practices in their classrooms while researchers may find research stimuli for further research activity.

Thank you,

Dr Isaak Papadopoulos

Guest Editor - Interculturalism in Second Language Teaching 